\title{
Clinical significance of prostatic-urethral angulation on the treatment outcome of patients with symptomatic benign prostatic hyperplasia treated with tamsulosin hydrochloride
}

\author{
Hassan El-Tatawy ${ }^{1}$, Tarek Gameel ${ }^{1}$, Mohammed Abo El-enen ${ }^{1}$, Ayman Hagras ${ }^{1}$, Ayman Mousa ${ }^{1}$, \\ Abdel Hamid El-Bahnasy ${ }^{1}$, Ali Abdel Raheem ${ }^{1}$, Khaled Abu-dewan ${ }^{2}$ \\ ${ }^{1}$ Urology Department, Tanta University Hospital, Egypt. \\ ${ }^{2}$ Radiology Department, Tanta University Hospital, Egypt.
}

\begin{abstract}
Summary Objectives: To evaluate the impact of the prostatic-urethral angulation (PUA) on the treatment efficacy of selective alpha-1A receptor blocker in male patients with lower urinary tract symptoms secondary to benign prostatic hyperplasia (LUTS/BPH).

Materials and methods: A total of 80 patients with LUTS/BPH and with mean age $53.3 \pm 6.3$ (range 47-70) were included in our prospective comparative study. The patients were classified into 2 groups as a consecutive cases 40 in each one depending on the PUA either $\leq 35^{\circ}$ (group A) or $>35^{\circ}$ (group $B)$. PUA and different prostatic parameters were measured using transrectal ultrasound. Prostate-specific antigen (PSA), the International Prostate Symptom Score and quality of life score (IPSS/QoL score), maximum flow rate $\left(Q_{\text {max }}\right)$, and postvoid residual (PVR) volume were compared between the groups. The clinical significance of PUA was evaluated after 8 weeks of medical treatment with tamsulosin hydrochloride $0.4 \mathrm{mg}$ daily.

Results: Baseline evaluation (pre-treatment) for both groups were comparable to each other with no clinically significant difference regarding age, PSA, IPSS/QoL score, $\mathcal{Q}_{\max }$ and PVR volume $(P$-value $>0.05)$. Comparison of parameters after 8 weeks showed that tamsulosin hydrochloride improved the total IPSS and all subscores $(P<0.001)$, QoL $(P=0.001)$, $Q_{\max }(P=0.002)$, and PVR $(P=0.04)$ in group A (Table 1$)$. Conclusion: Tamsulosin hydrochloride appears to be less effective in improving IPSS/Qol score, Qmax and PVR in patients with lager PUA. The PUA might be a predictor for the treatment efficacy of $\alpha$-blockers and more studies are warranted in the future before the final conclusion.
\end{abstract}

KEY WORDS: Prostatic-urethral angulation, benign prostatic hyperplasia and tamsulosin hydrochloride.

Submitted 20 February 2015; Accepted 30 April 2015

\section{INTRODUCTION}

Benign prostatic hyperplasia (BPH) is one of the most common health problems affecting the aging males. It affects more than 200 million males globally (1). Lower urinary tract symptoms (LUTS) secondary to BPH (LUTS/BPH) are bothersome symptoms which have a negative impact on men's quality of life (QoL).
Up till now the exact pathophysiology of BPH is still poorly understood and different theories have emerged and changed over the past years. Some studies reported that the prostate volume does not correlate with the clinical LUTS and/or the degree of bladder outlet obstruction (BOO) $(2,3)$, and other studies discussed several anatomical factors that may explain the clinical effect of BPH such as intravesical prostatic protrusion (IPP), transition zone volume (TZV), transition zone index (TZI) and presumed circle area ratio (4-9).

Recently, Cho et al. introduced the term prostatic-urethral angulation (PUA) as a new measurement that could be a causal factor for BPH (10), and in their subsequent preliminary clinical study using the fluid dynamic model, they reported that the urinary flow rate decreased by more than $27 \%$ as the PUA increased from $35^{\circ}$ to $90^{\circ}$ (11). Patients with PUA $\geq 35^{\circ}$ had larger prostate volume and higher $\mathrm{BOO}$ index, in comparison with those who had PUA $<35^{\circ}$ (12). Further studies showed that the PUA is significantly associated with maximum flow rate (Qmax) and voiding symptom scores in men with LUTS (13-14). In terms of medical therapy for BPH several drugs are widely distributed in the market and selective $\alpha 1$-blocker is the recommended treatment (15). Tamsulosin hydrochloride is a highly selective $\alpha 1 \mathrm{~A}$-blocker that is currently used and proved to be effective and safe in treatment of symptomatic $\mathrm{BPH}$ alone or in combination with other drugs (16).

The effect of PUA on the treatment outcome of tamsulosin hydrochloride on men with LUTS was first evaluated and PUA was inversely correlated with changes in Qmax and symptoms score after treatment (17).

The aim of the present study is to evaluate the clinical outcome of medical treatment (by selective $\alpha$ lA-blocker) on male patients presented with LUTS/BPH and its correlation to the degree of PUA.

\section{Patients And methods}

This prospective comparative study was conducted at our outpatient clinic of the Urology Department in the 
period between October 2012 and October 2014. The study was approved from our ethical committee and an informed consent was taken from all patients before the start of the study.

We included male patients aged $\geq 40$ years and $<70$ years who presented with LUTS/BPH for at least 6 months and who did not receive any medical treatment for BPH before. We excluded patients with suspicious hypersensitivity to alpha blockers, a disease causing LUTS such as (urinary calculi, urethral stricture, urinary tract infection, primary renal disease, neurogenic bladder, prostatitis), associated co-morbidities such as uncontrolled diabetes mellitus, a history of bladder or prostate carcinoma, previous lower urinary tract surgery, renal impairment, serum PSA $\geq 4 \mathrm{ng} / \mathrm{ml}$ and a post-void residual (PVR) urine volume $>150 \mathrm{ml}$.

All patients were evaluated through detailed history, full physical examination including digital rectal examination and neurological examination. The International Prostate Symptom Score and quality of life score (IPSS/QoL score) were estimated for each patient and IPSS score was subdivided according to storage symptoms subscore and voiding symptoms subscore. Laboratory investigations included urine analysis, fasting blood glucose level, serum creatinine and serum prostate-specific antigen (PSA).

All patients underwent transrectal ultrasound by the same radiologist to assess the degree of PUA and total prostate volume (TPV). We used GeVoluson E8 ultrasound machine (8 MHz, General Electric Company, USA), the patient was placed in right sided raised position and anal dilatation was done before insertion of the rectal probe. We acquired B-mode picture to locate the longitudinal section for the prostate and choose the generic angle and caliber lists (from the keyboard of the apparatus). A line was drawn passing longitudinally through the proximal part of prostatic urethra and another line passing longitudinally through the distal part of prostatic urethra. The angle between the two lines represents the PUA (Figure 1). The TPV was determined by measuring the length, height, and width of the whole prostate in both transverse and axial sections using the formula (length $\times$ width $\times$ height $\times 0.52$ ). Uroflowmetry was performed for all patients (at least 2 voids with minimum voided volume $=150 \mathrm{ml}$ ), PVR urine was measured using bladder scan after micturition using the formula (length $\times$ width $\times$ height $\times 0.7$ ).

Patients were classified into two groups as consecutive cases according to the PUA: group A (patients with PUA $\leq 35^{\circ}$ ) and group B (patient with PUA $>35^{\circ}$ ). The PUA cutoff value in previous studies was $35^{\circ}$; at this angle a significant difference in clinical symptoms and urodynamics parameters in patients and healthy men were found. Subsequently, we classified our patients into 2 categories (PUA $\leq 35^{\circ}$ and $\mathrm{PUA}>35^{\circ}$ ) based on these studies (11-12).

All patients received medical treatment in the form of tamsulosin hydrochloride $0.4 \mathrm{mg}$ once daily for 8 weeks. The clinical significance of PUA was evaluated subjectively by measuring the IPSS/QoL score and objectively by measuring uroflowmetry parameters $\left(\mathrm{Q}_{\max }\right.$ and PVR volume).

Statistical analysis was performed using SPSS ${ }^{\circledR} 17$. Data are shown as the mean \pm SD unless otherwise specified. The Student $t$ and paired sample $t$ tests were used for comparison between groups and in the same group, respectively. Statistical significance was considered at $\mathrm{p}<0.05$.

\section{RESULTS}

Eighty patients were included in our study, 40 in each group. Two patients were excluded as they were lost during follow-up (1 patient in each group). Patients mean age was $53.3 \pm 6.3$ (range 47-70). Mean IPSS score, QoL score; $\mathrm{Q}_{\max }, \mathrm{PVR}$ and TPV for all patients were $16.8 \pm$ $2.5,3.43 \pm 0.9,9.9 \pm 1.5,62.4 \pm 12.2$ and $57.3 \pm 11.9$ respectively.

Baseline evaluation data (pre-treatment) for both groups were comparable to each other with no clinically significant difference regarding age, PSA, IPSS/QoL score, uroflometry and IPP (P-value > 0.05). On the contrary, patients in group $\mathrm{A}$ had a smaller prostate than those in group B with mean TPV $49.54 \pm 9.03$ and TZV $11.6 \pm$ $1.4(p$-value $=0.001$ and 0.001 , respectively) (Table 1).

\section{Figure 1.}

Ultrasound image used in measurement for PUA in two patients of both groups (Lt. Photo: PUA $=32.3^{\circ}$, Rt. Photo: PUA $=44.4^{\circ}$ ).

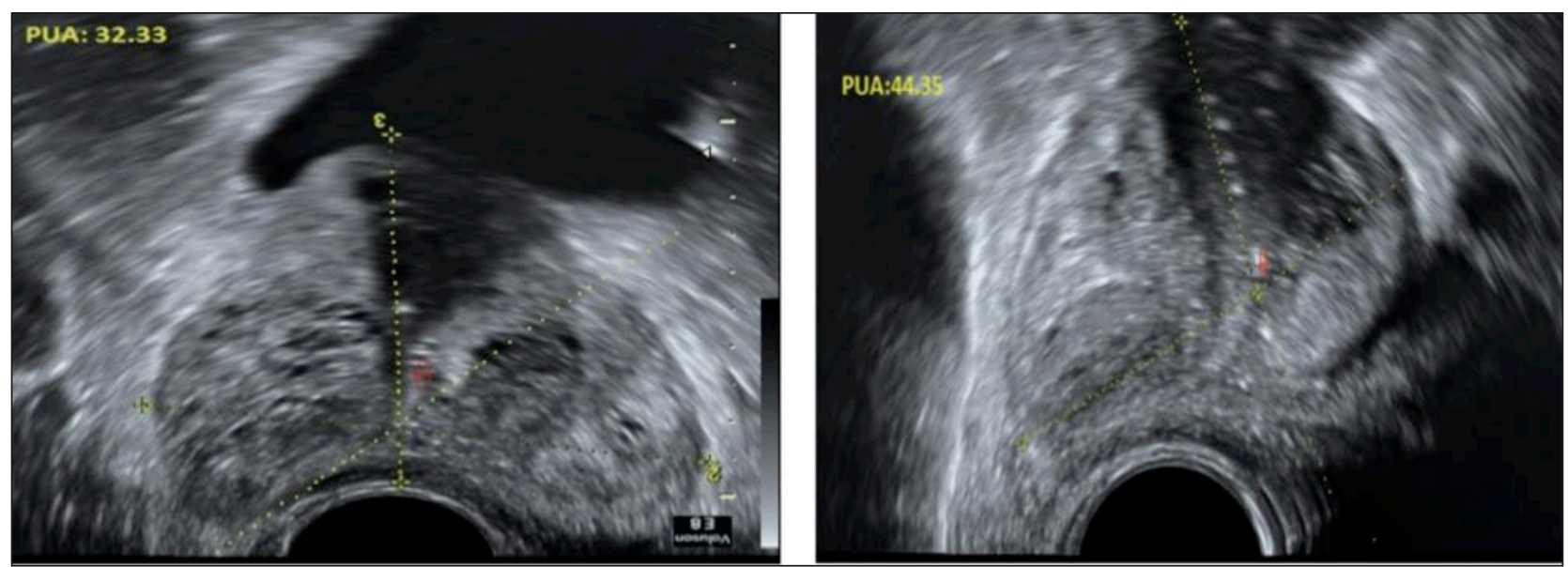


Table 1.

Patients characteristics (pretreatment data) in both groups.

\begin{tabular}{|lccc|}
\hline & $\begin{array}{c}\text { Group A } \\
\text { Mean } \pm \text { SD }\end{array}$ & $\begin{array}{c}\text { Group B } \\
\text { Mean } \pm \text { SD }\end{array}$ & P-value \\
\hline PUA & $\leq 35^{\circ}$ & $>35^{\circ}$ & - \\
\hline Patients number & 40 & 40 & - \\
\hline Age (yr) & $55.41 \pm 9.03$ & $58.23 \pm 6.37$ & 0.78 \\
\hline PSA (ng/ml) & $2.4 \pm 1.13$ & $2.4 \pm 1.39$ & 0.83 \\
\hline Prostatic measurements: & & & \\
- TPV (cm ${ }^{3}$ ) & $49.54 \pm 9.03$ & $62.32 \pm 11.99$ & $\mathbf{0 . 0 0 1}$ \\
- IPP (mm) & $3.5 \pm 1.1$ & $3.7 \pm 0.8$ & 0.19 \\
- TZV (cm $\left.{ }^{3}\right)$ & $11.6 \pm 1.4$ & $19.5 \pm 1.3$ & $\mathbf{0 . 0 0 1}$ \\
\hline Uroflometry data: & & & \\
Qmax (ml/s) & $9.75 \pm 1.34$ & $10.37 \pm 1.43$ & 0.45 \\
PVR (ml) & $69.66 \pm 10.49$ & $66.12 \pm 11.76$ & 0.09 \\
Voided volume (ml) & $185.9 \pm 11.5$ & $198.72 \pm 17.51$ & 0.43 \\
\hline Overall IPSS score: & $17.95 \pm 3.61$ & $15.54 \pm 3.53$ & 0.68 \\
- Voiding symptoms & $7.84 \pm 2.2$ & $6.88 \pm 1.72$ & 0.06 \\
- Storage symptoms & $8.48 \pm 2.33$ & $7.35 \pm 1.64$ & 0.13 \\
- Bother score (QoL) & $3.1 \pm 1.79$ & $3.37 \pm 0.96$ & 0.09 \\
\hline
\end{tabular}

After 8 weeks of follow-up of medication with tamsulosin hydrochloride there was a significant improvement in IPSS/QoL score, $\mathrm{Q}_{\max }$ and PVR urine in group A (pvalue $=0.001,0.001,0.002$ and 0.04 respectively). On the other hand, there was no significant improvement in the above mentioned parameters in group B (Table 2). On comparison between both groups there was a statistically significant improvement in group A in IPSS/QoL score, Qmax and PVR volume compared to group B (pvalue $=0.001,0.03,0.01,0.05$ and 0.002 , respectively) (Table 2).

There were no moderate or severe side effects in either group lead to discontinuation of medical treatment. In our study, the most common adverse event was dizziness in $7.6 \%$ of patients ( 3 in each group), while the abnormal ejaculation rates were $5.1 \%$ in group A and $7.7 \%$ in group B.

\section{Discussion}

Several anatomical factors of the prostate other that its volume were studied in the past years to assess its impact on the clinical symptoms progression such as IPP, TZI,
TZV and presumed circle area ratio (2-9). )Another recent anatomical factor was introduced by Cho et al. and it might play a role in the pathogenesis of $\mathrm{PBH}$ is the PUA. The prostatic urethra is a bent tube and by applying the concept of fluid dynamics to the micturition process in the prostatic urethra, they hypothesized that some energy loss could occur during this process and hence decreasing the velocity of urine. In addition, they suggested that this energy loss increased proportionally as the PUA increased and $Q_{\max }$ decreased by more than $27 \%$ as the PUA increased from $35^{\circ}$ to $90^{\circ}$ (10-11).

The present study is the first prospective comparative study that addresses the importance of the PUA as a predictor of the clinical status and treatment outcome in patients with LUTS/BPH who received selective $\alpha 1$ Ablockers. As we know $\alpha$-blockers help to treat BPH by relaxation of the smooth-muscle tissue in the bladder neck and the prostate, which facilitate urinary outflow from the bladder more easily. We chose tamsulosin hydrochloride in our study because it is a highly selective $\alpha 1 \mathrm{~A}$-blocker, safe and has long-term treatment efficacy $(15,16,18)$.

In the present study, baseline age, PSA, IPP, uroflowmetry, IPSS/QoL score did not differ significantly between the two groups. However, TPV and TZV did differ significantly between the groups at baseline $(\mathrm{P}<0.05)$. TPV and TZV were higher in patients with PUA $>35^{\circ} \mathrm{com}$ pared to those with lower PUA and these findings were in accordance to Ku et al. who showed that patients with PUA $>35$ have higher prostate volume than those with PUA $\leq 35$ (12)

Our study showed no significant difference in Qmax values in the two groups of patients (p-value $=0.45)$ (Table 1). In contrast to our findings, the urinary flow rate was found inversely associated with the PUA (11), and a larger PUA was associated with a lower urinary flow rate (14). On the other hand, no significant difference in Qmax values between patients with PUA more or less $35^{\circ}$ was reported (12). The reason for this difference between our study and others may be the selection of our patients. In fact we excluded all patients with insignificant symptoms related to their enlarged prostate. Consequently, the pre-treatment symptoms and related objective signs are expected to be matching. In contrary, Cho et al. (11) did not choose their patients on such

\section{Table 2.}

Intragroup and intergroup comparisons of subjective (IPSS score) and objective (uroflometry) parameters after 2 months of treatment with tamsulosin hydrochloride.

\begin{tabular}{|c|c|c|c|c|c|c|c|}
\hline & \multicolumn{3}{|c|}{$\begin{array}{c}\text { Group A } \\
\text { Mean } \pm \text { SD }\end{array}$} & \multicolumn{3}{|c|}{$\begin{array}{c}\text { Group B } \\
\text { Mean } \pm \text { SD }\end{array}$} & \multirow[b]{2}{*}{$\begin{array}{l}\text { Post-treatment } \\
\text { P-value }\end{array}$} \\
\hline $\begin{array}{l}\text { Subjective/objective } \\
\text { parameters }\end{array}$ & Baseline & After treatment & P-value & Baseline & After treatment & P-value & \\
\hline Total IPSS score: & $17.95 \pm 3.61$ & $11.85 \pm 2.43$ & 0.000 & $15.54 \pm 3.53$ & $14.34 \pm 3.71$ & 0.9 & 0.001 \\
\hline - Voiding symptoms & $7.84 \pm 2.2$ & $5.93 \pm 2.12$ & 0.001 & $6.88 \pm 1.72$ & $6.10 \pm 1.43$ & 0.27 & 0.03 \\
\hline - Storage symptoms & $8.48 \pm 2.33$ & $5.95 \pm 1.89$ & 0.001 & $7.35 \pm 1.64$ & $5.53 \pm 1.79$ & 0.03 & 0.01 \\
\hline - QoL score & $3.17 \pm 1.79$ & $1.97 \pm 1.82$ & 0.0011 & $3.37 \pm 0.96$ & $3.12 \pm 1.20$ & 0.42 & 0.006 \\
\hline \multicolumn{8}{|l|}{ Uroflometry: } \\
\hline $\mathrm{Q}_{\max }(\mathrm{ml} / \mathrm{s})$ & $9.75 \pm 1.34$ & $12.95 \pm 1.72$ & 0.002 & $10.37 \pm 1.43$ & $11.10 \pm 1.56$ & 0.06 & 0.05 \\
\hline PVR (ml) & $69.66 \pm 10.49$ & $58.98 \pm 12.64$ & 0.04 & $66.12 \pm 11.76$ & $61.12 \pm 12.63$ & 0.32 & 0.002 \\
\hline
\end{tabular}


basis, that is symptomatic patients who require treatment. In their study the patients were healthy men with no evidence of $\mathrm{BOO}$ (patients age 50 to 59 years with prostate volume $30 \mathrm{ml}$ and $\mathrm{Q}_{\max } 15 \mathrm{ml} / \mathrm{s}$ ). Moreover, the large number of patients in these studies may help to make clear explanations. After 2 months of medical treatment, a significant improvement in $\mathrm{Q}_{\max }$ and PVR urine was found in group A (p-value $=0.000$ and 0.04 ), while no improvement was present in group B ( $\mathrm{p}$-value $=0.9$ and 0.32) (Table 2). More recently, it was found that the improvement in $Q_{\max }$ values from baseline after 3 months of tamsulosin therapy was poor in patients with higher PUA, and this is evident in our study (17).

It is well known to us that the IPSS/Qol score is an essential subjective tool for assessing symptoms severity and disease-specific QoL when determining treatment strategies and evaluating treatment outcome in men with LUTS/BPH (19). We utilized the IPSS/QoL score in the subjective evaluation of medical treatment outcome in our patients. At baseline evaluation (pre-treatment), no difference in the IPSS/QoL score was found between both groups $(\mathrm{P}$-value $=0.68$ and 0.09 respectively) $($ Table 1$)$. These findings are similar and agree with previous reports (12-13). At 2 months of follow-up our study showed a statistically significant improvement in the IPSS/QoL scores in group A compared to group B (P-value $=0.0005$ and $<0.000$ respectively) (Table 2 ). This can be explained by the presence of significant mechanical factors for obstruction among patients in group B (PUA > 35). In presence of this factor it would be expected no response to alpha blocker drugs that usually affect the dynamic part of the obstruction. Our findings were similar to those of Hou et al. who investigated the association of the PUA and the severity of LUTS on the aging male. They also evaluated the PUA effect on tamsulosin hydrochloride therapy: after 3 months of medical treatment they found that the PUA had an extremely strong correlation with IPSS score and was inversely correlated with changes in the IPSS score after tamsulosin treatment (17).

To our knowledge, the prostatic urethra passes through the prostate from its base to its apex and this lead to formation of an anterior angle of $35^{\circ}$ proximal to the veru montanum, this angle divides the urethra into proximal and distal regions (20). In men with nodular hyperplasia the angle tends to be $>35^{\circ}$, however, it could be also increased in men without nodular hyperplasia, as found during cystoscopic examination; an increased PUA, results in a higher bladder neck without lateral or median lobe enlargement which is suggested as a clinically significant causal factor of male LUTS (10-11).

Looking at measurement of the prostatic parameters in our patients, the TPV and TZV were higher in patients with PUA $>35^{\circ}$ and the IPP was similar with no significant difference between both groups. In fact several studies reported that the prostate volume does not correlate with the clinical LUTS and/or the degree of $\mathrm{BOO}(2,3)$; in addition the mean IPP of our patients was small $3.6 \pm$ $1.1 \mathrm{~mm}$, while Keqin et al. used an IPP cutoff value of 10 $\mathrm{mm}$ for better classification of $\mathrm{BOO}$ in patients and correlation with clinical symptoms (21). Therefore, it is clearly evident that the PUA is an important prostatic measurement and it might be a predictor of treatment efficacy of $\alpha$-blockers in men with LUTS/BPH. Medical treatment use for treating symptomatic BPH patients may result in a delay in the surgical intervention decision. Consequently, patients usually become older, may become unfit for surgery, and may have advanced disease; this may result in unfavorable outcomes and more postoperative morbidities and complications. Also, delay in surgical treatment results in more serious and complicated resection (22-23). For this reasons, a predictive factor to those patients who will fail medical treatment in the future can guide us to spend less money as well as to avoid possible complications for such patients that can treated earlier by definitive surgery rather than remaim on medications for some time before going to surgery. The result of our study revealed that patients with higher PUA $>35^{\circ}$ showed a poor response to $0.4 \mathrm{mg}$ tamsulosin hydrochloride in both subjective and objective parameters than those with PUA $\leq 35^{\circ}$. In another recent study, authors reported that patients with PUA $<48.3^{\circ}$, did not require transurethral resection of the prostate (TURP) within 1 year of medical treatment. While, 19 patients of those with PUA $>48.3^{\circ}$ finally received TURP within 1 year due to lower tamsulosin hydrochloride efficacy (17).

There are some limitations in our study as the small number of patients each group, the short term follow-up and the selection bias for patients during classification in groups based on previous cutoff values of PUA in other studies.

Looking at the objective as well as the subjective data at the present study, we are suggesting that the PUA may have an important role in the symptomatology of the patients with LUTS/BPH. Also, based on the present data of little response to medical treatment among those patients with higher PUA $>35$, we suggest that patients with higher PUA should be offered another line of treatment and )informed explaining the expected results.

\section{Conclusions}

In conclusion, this preliminary study suggests that the PUA appears to be one of the anatomical factors which has an impact on medical treatment of male patients with LUTS/BPH and it might be a predictor for the treatment efficacy of $\boldsymbol{\alpha}$-blockers. More studies are warranted in the future before drawing the final conclusion. These studies should include larger numbers of patients with longer follow-up periods and should be focused on the PUA and especially on the method of its measurement and the relationship to the symptoms as well as its effect on every available methods of treatment (medical, minimally invasive, or invasive).

\section{References}

1. Verhamme KM, Dieleman JP, Bleumink GS, et al. Incidence and prevalence of lower urinary tract symptoms suggestive of benign prostatic hyperplasia in primary care - the Triumph project. Eur Urol. 2002; 42:323-328.

2. Bushman W. Etiology, epidemiology, and natural history. Urol Clin North Am. 2009; 36:403-415. 
3. Sciarra A, D'Eramo G, Casale P, et al.Relationship among symptom score, prostate volume, and urinary flow rates in 543 patients with and without benign prostatic hyperplasia. Prostate 1998; 34:121-128.

4. Kaplan SA, Te AE, Pressler LB, et al. Transition zone index as a method of assessing benign prostatic hyperplasia: correlation with symptoms, urine flow and detrusor pressure. J Urol. 1995; 154:1764-9.

5. Lepor H, Nieder A, Feser J, et al. Total prostate and transition zone volumes, and transition zone index are poorly correlated with objective measures of clinical benign prostatic hyperplasia. J Urol. 1997; 158:85-8.

6. Chia SJ, Heng CT, Chan SP, et al. Correlation of intravesical prostatic protrusion with bladder outlet obstruction. BJU Int. 2003; 91:371-4.

7. Keqin Z, Zhishun X, Jing Z, et al. Clinical significance of intravesical prostatic protrusion in patients with benign prostatic enlargement. Urology 2007; 70:1096-1099.

8. Kojima M, Ochiai A, Naya Y, et al. Correlation of presumed circle area ratio with infravesical obstruction in men with lower urinary tract symptoms. Urology 1997; 50:548-555.

9. Doo CK and Uh. HS. Anatomic configuration of prostate obtained by noninvasive ultrasonography can predict clinical voiding parameters for determining BOO in men with LUTS. Urology. 2009; 73:232-236.

10. Cho KS, Kim J, Choi YD, et al. The overlooked cause of benign prostatic hyperplasia: prostatic urethral angulation. Med Hypotheses. 2008; 70:532-535.

11. Cho KS, Kim JH, Kim DJ, et al. Relationship between prostatic urethral angle and urinary flow rate: its implication in benign prostatic hyperplasia pathogenesis. Urology. 2008; 71:858-862.

12. Ku JH, Ko DW, Cho JY, et al. Correlation between prostatic urethral angle and bladder outlet obstruction index in patients with lower urinary tract symptoms. Urology. 2010; 75:1467-1471.

13. Park YJ, Bae KH, Jin BS, et al. Is Increased Prostatic Urethral Angle Related to Lower Urinary Tract Symptoms in Males with
Benign Prostatic Hyperplasia/Lower Urinary Tract Symptoms? Korean J Urol. 2012; 53:410-413.

14. Bang WJ, Kim HW, Leec JY, et al. Prostatic Urethral Angulation Associated With Urinary Flow Rate and Urinary Symptom Scores in Men With Lower Urinary Tract Symptoms. Urology. 2012; 80:1333-1337.

15. Gravas S, Bachmann A, Descazeaud A, et al. Guidelines on the Treatment of Non-neurogenic Male LUTS including benign prostatic obstruction. European Association of Urology, 2014.

16. Roehrborn CG, Barkin J, Tubaro A, et al. Influence of baseline variables on changes in International Prostate Symptom Score after combined therapy with dutasteride plus tamsulosin or either monotherapy in patients with benign prostatic hyperplasia and lower urinary tract symptoms: 4-year results of the CombAT study. BJU Int. 2014; 113:623-35.

17. Houl C, Chen L, Lin Y, et al. Prostatic urethral angle might be a predictor of treatment efficacy of $\alpha$-blockers in men with lower urinary tract symptoms. Drug Design, Development and Therapy. 2014:8 937-943.

18. Narayan P, Lepor H. Long-term, open-label, phase III multicenter study of tamsulosin in benign prostatic hyperplasia. Urology. $2001 ; 57: 466-470$

19. Yoshida M, Sugiyama Y, Masunaga K, et al. Effect of tamsulosin hydrochloride on lower urinary tract symptoms and quality of life in patients with benign prostatic hyperplasia. Evaluation using bother score. Drugs Today. 2007; 43:1-7.

20. McNeal JE. The prostate and prostatic urethra: a morphologic synthesis. J Urol. 1972; 107:1008-1016.

21. Keqin Z, Zhishun X, Jing Z, et al. Clinical significance of intravesical prostatic protrusion in patients with benign prostatic enlargement. Urology. 2007; 70:1096-1099.

22. Borth CS, Beiko DT, Nickel JC. Impact of medical therapy on transurethral resection of the prostate: a decade of change. Urology. 2001; 57:1082-5.

23. Jepsen JV, Bruskewitz RC. Recent developments in the surgical management of benign prostatic hyperplasia.. Urology 1998; 51:23-31.

\section{Correspondence}

Ali Abdel Raheem, MD (Corresponding Author)

aliraheem82@yahoo.com

Hassan El-Tatawy

hassantat@yahoo.com

Tarek Gameel

t_gameel@yahoo.com

Mohammed Abo El-enen

m.aboelenen@yahoo.com

Ayman Hagras

aymanhagras@yahoo.com

Ayman Mousa

hn.hn2020@yahoo.com

Abdel Hamid El-Bahnasy

mrabdo65@yahoo.com

Urology Department, Tanta University Hospital, Egypt

Khaled Abu-dewan

Radiology Department, Tanta University Hospital, Egypt 